\title{
Research on cluster overlap and non-overlap region for 5G-loV networks with NOMA
}

\author{
Jinyuan Gu ${ }^{1,2}$, Guoan Zhang ${ }^{1 *}$, Haibin Lv ${ }^{3}$ and Wei Duan ${ }^{1}$
}

\section{*Correspondence:}

gzhang@ntu.edu.cn

${ }^{1}$ School of Information

Science and Technology,

Nantong University,

Nantong 226000, China

Full list of author information

is available at the end of the

article

\begin{abstract}
As a multi hop self-organizing network, wireless sensor network has the ability to cooperatively sense, collect and process the information of the sensed objects. The applications of WCN in 5G-based Internet of Vehicles (5G-loV), using information fusion and intelligent information processing technologies, can obtain more reliable and accurate detection parameters, which has been widely concerned. However, the massive connectivity and information exchange in 5G-loV pose great challenges to the bandwidth efficiency. In order to overcome these issues in 5G-loV networks, a performance enhanced scheme based on non-orthogonal multiple access (NOMA) is proposed. In the proposed scheme, different vehicle locations are respectively discussed, i.e., whether in the overlap region of cluster head vehicles (CHVs). In particular, different to conventional works, each receiving node only decodes the desired signal to avoid performance loss provided from the poor channel quality limitation. On the other hand, all CHVs decode-and-forward new superposition coded signals with new power allocation factors, while that the maximum ratio combining is utilized at receivers to further improve the ergodic sum-rate (SR) and probability of conflict. The closed-form expressions of ergodic SR for our proposed scheme are analyzed under the independent Rayleigh fading channels. Numerical results corroborating our theoretical analysis show that the superposition coded signal transmission scheme applied to the proposed NOMA-loV improves the ergodic SR performance significantly compared with the existing works, especially for the high signal-to-noise region.
\end{abstract}

Keywords: Wireless sensor network (WSN), Non-orthogonal multiple access (NOMA), 5G-Internet of Vehicles (IoV), Ergodic sum-rate, Cluster overlap region

\section{Introduction}

In recent years, traffic safety, travel efficiency, environmental protection and other issues caused by the growing number of cars have became particularly prominent, which results in the research and development of related fields of Internet of Vehicles (IoVs) concerning [1,2]. The IoV is based on the intranet and mobile Internet, which integrates the Global Position System (GPS), sensors, radio frequency identification (RFID), data mining, automatic control and other related technologies [3]. According to the agreed architecture, communication protocol and data interaction standard, it is in the process of the vehicle- $\mathrm{X}(\mathrm{X}$ : vehicle, road, pedestrian, Internet) interaction to realize the integrated network of intelligent traffic management, intelligent dynamic information

c) The Author(s) 2021. Open Access This article is licensed under a Creative Commons Attribution 4.0 International License, which permits use, sharing, adaptation, distribution and reproduction in any medium or format, as long as you give appropriate credit to the original author(s) and the source, provide a link to the Creative Commons licence, and indicate if changes were made. The images or other third party material in this article are included in the article's Creative Commons licence, unless indicated otherwise in a credit line to the material. If material is not included in the article's Creative Commons licence and your intended use is not permitted by statutory regulation or exceeds the permitted use, you will need to obtain permission directly from the copyright holder. To view a copy of this licence, visit http:// creativecommons.org/licenses/by/4.0/. 
service and vehicle intelligent control. Moreover, the IoV is a typical application of the Internet of Things (IoT) [4] technology in the field of transportation system with the core as Vehicle to Everything (V2X) wireless communication technology [5, 6]. With the help of V2X wireless communication technology, IoVs can not only break through the technical bottleneck of single vehicle in intelligent development, but also intuitively improve the intelligent level and automatic driving ability of the Internet connected vehicles [7]. In particular, V2X provides the traffic efficiency enhancement, driving experience improvement, the new business form of the intelligent and comfortable, safe, energysaving and efficient comprehensive services. In addition, the mainstream technology of V2X communication includes Dedicated Short Range Communication (DSRC) technology [8], and Cellular V2X (C-V2X) technology based on the cellular mobile communication system. Currently, the C-V2X technology is mainly based on LTE-V2X technology [9-11], which evolves to the 5th generation mobile communication (5G)-based V2X technology in the future. Specifically, the vehicle clustering has been utilized to reduce the complexity of V2X communications that would ultimately improve road traffic efficiency.

On the other hand, with the rapid development of 5G, in the face of the explosive growth of network demands in the future wireless communications, $5 \mathrm{G}$ mobile network considers the wireless transmission technologies that can further explore the potential of spectrum efficiency improvement, i.e., large-scale antenna technology, millimeter wave communication, ultra-density network, non-orthogonal multiple access, etc. [12]. In order to meet the massive access and ultra-high capacity demands of $5 \mathrm{G}$ network, non-orthogonal multiple access (NOMA) $[13,14]$ technology is considered as the most critical technology in the next generation of mobile communication. Based on the multiuser information theory, NOMA technology can further improve the number of users' connections by introducing new dimensions such as power and code domains with the existing physical resources, providing more channel capacity than the conventional orthogonal multiple access (OMA) [15-17].

It is known that NOMA technology can not only meet the requirements of higher spectrum efficiency, but also satisfy the user fairness condition. Therefore, it is serving as a potential technology for the future beyond $5 \mathrm{G}(5 \mathrm{~GB})$ and the 6 th generation wireless communication (6G) system $[18,19]$. Therefore, the combination of NOMA technology in vehicle network enables multiple vehicles to transmit information by sharing the same channel simultaneously, thus alleviating resource conflicts such as limited transmission capacity and unpredictable transmission delay in 5G-V2X communications. The basic idea of NOMA is to use non-orthogonal transmission at the transmitter, introduce interference information actively and realize correct demodulation at the receiver via the successive interference cancellation (SIC). Even that the receiver using SIC technology is more complex, it can achieve a higher spectrum efficiency. In fact, the core idea of NOMA technology is to increase spectrum efficiency at the acceptable cost of receiver complexity [20]. With the in-depth study of 5G and continuous improvement of chip processing capacity, the processing complexity of the receiver has significantly improved, which leads to that NOMA technology inevitably becomes a hot topic in academia.

With above observations, in this paper, NOMA is introduced to the 5G-IoV networks with two adjacent cluster head vehicles (CHVs) to satisfy the spectrum efficiency and 
massive connectivities requirements. Particularly, since the overall performance of the system is limited by the poor channel [21], at the CHVs, only the desired signals for the vehicles will be decoded and forwarded. We further discuss the effects of different vehicle locations: (1) the vehicle is in the overlap region of the CHVs; (2) the vehicle is not in the overlap region of the CHVs, which means that multiple CHVs can support the service or not. For our proposed NOMA-IoV networks, all the CHVs will forward the new superposition coded signals to the vehicles in the served region to further improve the ergodic sum-rate (SR) of the system. These motivate us to investigate the proposed NOMA-IoV system with with multiple CHVs. The following are the key contributions of this paper:

- In order to further improve the ergodic SR, we comprehensively investigate the NOMA-IoV networks with two CHVS. Different to the conventional NOMA scheme, after receptions, the CHVs only decode-and-forward (DF) the desired signals to the vehicles, in the meanwhile, the maximum ratio combining (MRC) is also utilized to improve the performance. To the best of our knowledge, combining NOMA-V2X networks with overlap region discussion has not been considered so far, which is more general and challenging.

- The closed-form solutions of the ergodic SR for the proposed schemes at the high transmit signal-to-noise ratio (SNR) region are derived with a negligible performance loss. It is worth noting that, there are few works that focus on the 2-stage superposed transmission for multiple CHVs NOMA-V2X networks, since it is hard to obtain the exact expression of the ergodic SR. Furthermore, the theoretical results are shown to highly agree with the simulation results, especially in the high SNR region.

- By means of numerical results, both analytically and numerically, we compare the proposed NOMA-IoV schemes with the TDMA scheme in terms of ergodic SR. It is shown that the proposed NOMA-IoV with the overlap region service scheme outperforms the TDMA and existing NOMA schemes significantly.

The rest of this paper is organized as follows. Related work is first introduced in Sect. 2. In Sect. 3, we present the proposed model and corresponding problems. The performance of proposed NOMA-IoV in terms of the ergodic SR is analyzed in Sect. 4 including the overlap, non-overlap and conventional NOMA schemes. Section 5 contains the system simulation conditions and results. This paper is concluded in Sect. 6.

\section{Related work}

In recent years, adopting NOMA in vehicle network has became a popular research hotspot. In [22], the author studied the potential capacity of NOMA in the V2X scenario and difficulties in the next generation communication. From the unicast system, a spectrum resource allocation scheme based on NOMA is proposed, which is extended to the broadcast system. Based on this, the authors in [23] investigate the key problems of interference management and handover in $5 \mathrm{G}$ vehicle communication system with NOMA. Moreover, in order to improve the spectrum efficiency of 5G vehicle communication system based on NOMA and adapt to traffic load conditions and high mobility, a hierarchical power control scheme is proposed to realize joint optimization of cell 
correlation and power control. In [24], the power allocation of heterogeneous vehicle communication with NOMA under non-ideal channel estimation is studied, where the influence of channel estimation error caused by high mobility in vehicle communication on the realization of effective power distribution and link reliability is demonstrated. In addition, a NOMA-V2X network with spatial multiplexing permission is proposed in [25], which can be efficiently solved by a constructed interference hypergraph. However, the interference model in [25] only considers whether the interference exceeds a fixed threshold and ignores the impact of the interference level, which is difficult to reflect the real interference environment. It is known that, due to the real-time change of road traffic, the vehicle network is dynamic leading to the problem of network topology instability. According to this, in [26], a clustering method is proposed to make the network topology hierarchical and scalable by establishing the connectivity graph between the nodes of the vehicle network. To support data distribution and reduce the complexity of end-to-end (E2E) communication through the cluster head node, the authors in [27] investigate an efficient cluster selection scheme. This clustering method has been widely used in V2X communication to improve the performance of vehicle network [28, 29]. Since that the expansion of vehicle network leads to a huge increase in energy consumption, the power control is an effective way to reduce power consumption without reducing the connectivity and coverage of vehicle networks [30]. In order to balance the power allocation among the cluster heads and further improve the throughput of the downlink, the power control method is used in V2X communication. On the other hand, as mentioned above, NOMA has developed power domain multiplexing to meet the requirements of a higher overload transmission [22]. Based on this, some researchers have studied the power control scheme in 5G-IoV communication with NOMA [23, 31].

Clearly, the above discussed researches show that NOMA-enabled V2X communication will be a trend in the future, for its potential to improve spectrum efficiency, and the ability for the effective resource management. However, the interference management problems in the NOMA-aided vehicle communication scenario still need to be excavated, i.e., how to use vehicle clustering to reduce the complexity of V2X communication effectively to improve road traffic efficiency; how to balance the power allocation between CHVs; how to improve the downlink throughput with NOMA power control method. One can conclude that this will be a new and meaningful research direction to propose effective solutions to the above problems to improve the system performance.

\section{Methods}

In this paper, we focus on the downlink transfer scheme for NOMA-IoV system involving one base station (BS), two cluster head vehicles and three vehicles as shown in Fig. 1, where all the nodes are equipped with a single antenna with perfect channel state information (CSI) and half duplex transmission protocol. Two kinds of vehicles locations are considered, i.e., one is that the vehicle (V1) is located in the overlap region of two CHVs, the other is that the vehicle (V1) is not located in the overlap region. The other vehicles can only receive the signals from the corresponding CHVs. Let $h_{\mu}$, for $\mu \in\left\{B_{1}, B_{2},\left(v_{1}, U_{1}\right),\left(v_{1}, U_{2}\right),\left(v_{2}, U_{1},\right)\left(v_{2}, U_{3}\right)\right\}$, denote the independent Rayleigh fading channels from the BS to CHV1 and CHV2, from CHV1 to V1 and $\mathrm{V} 2$, and from CHV2 to V1 and V3, with the average powers as $\alpha_{B_{1}}, \alpha_{B_{2}}, \alpha_{v_{1}, U_{1}}, \alpha_{v_{1}, U_{2}}$, 


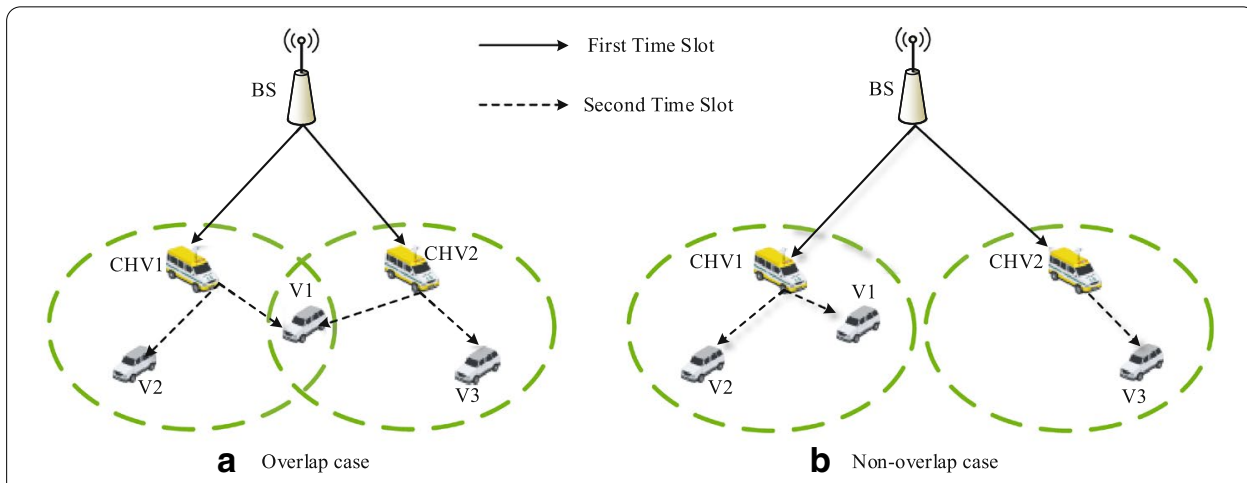

Fig. 1 The proposed NOMA-loV network: a overlap case, $\mathbf{b}$ non-overlap case

$\alpha_{v_{2}, U_{1}}$ and $\alpha_{v_{2}, U_{3}}$, respectively. We further assume that the two CHVs not only represent power domain differences between users, but also facilitate collaborative NOMA analysis and deployment. Therefore, the power distribution of two CHVs is sufficient to illustrate the key aspects of power distribution between vehicles, while avoiding unnecessary complications. Note that although we limit ourselves to two CHVS, the proposed approach can be easily extended to a multi-CHVs scenarios. It is also considered that the decode-and-forward (DF) scheme is adopted at each CHV.

It is worth noting that, due to the dense topology of the IoV network, when multiple CHVs (i.e., CHV1 and CHV2) are assigned the same time-frequency resource, those vehicle located in the overlap region of two adjacent CHVs has serious conflicts. In the traditional OMA case, a conflicting vehicle is likely to be unable to decode multiple CHV signals due to severe interference, resulting in additional retransmission delays. In order to reduce the probability of conflict and transmission delay, and consider the non-orthogonal allocation of wireless resources, a sub-channel can be simultaneously occupied by multiple CHVs. The data rate of the CHV-vehicle link can be increased compared to OMA schemes, and the vehicle for each collision is more likely to decode the received superposition coded signals using SIC technology. This can reduce the data conflicts and increase the number of users supported by the system.

With above descriptions, the superposition coded signal sent by the BS to these two CHVs can be denoted as

$$
\mathcal{S}=\sqrt{a_{1} P_{B}} x_{1}+\sqrt{a_{2} P_{B}} x_{2}+\sqrt{a_{3} P_{B}} x_{3}
$$

where $a_{i}$, for $i \in\{1,2,3\}$, is the power allocation coefficient with $a_{1}>a_{2}>a_{3}$ and $\sum_{1}^{3} a_{i}=1$, and $x_{i}$ is intended for the transmitted NOMA information at the BS satisfied $E\left[\left|x_{i}\right|^{2}\right]=1$. Moreover, we clarify that all the power allocation factors used in this paper are nonnegative. In our proposed scheme, each transmission involves two time slots. At the first time slot, the BS transmits the NOMA signal $\mathcal{S}$ to $\mathrm{CHVs}$, therefore, the received signals at $\mathrm{CHV} 1$ and $\mathrm{CHV} 2$ are respectively given by

$$
y_{B_{1}}^{(1)}=h_{B_{1}} \mathcal{S}+n_{1},
$$

and 


$$
y_{B_{2}}^{(1)}=h_{B_{2}} \mathcal{S}+n_{2},
$$

where $h_{B_{i}}$, for $i=\{1,2\}$, is the Rayleigh fading channel coefficient from BS to $i$-th CHV following the complex Gaussian distribution with the mean of zero and the variance of $\sigma_{B_{i}^{2}}$, i.e., $h_{B i} \sim \mathcal{C N}\left(0, \sigma_{B i}^{2}\right)$, and $n_{1}$ and $n_{2}$ are the AWGN with variance $N_{0}$ at CHV1 and $\mathrm{CHV} 2$, respectively. After receptions, $\mathrm{CHVs}$ will immediately decode the received superposition signal following the NOMA decoding principle, i.e., decode the symbol with higher power allocation factor first by treating the one with lower power allocation, and then cancel it to decode another one. Therefore, the received SINR for $x_{1}$ can be obtained from

$$
\gamma_{B_{j}}^{\left(x_{1}\right)}=\frac{\left|h_{B_{j}}\right|^{2} a_{1} \rho}{\left|h_{B_{j}}\right|^{2}\left(a_{2}+a_{3}\right) \rho+1}
$$

for $j \in\{1,2\}$, where $\rho=\frac{P_{B}}{N_{0}}$ denotes the transmit SNR at the BS. It is clear that the desired signals for the users in CHV1 served region are $\left\{x_{1}, x_{2}\right\}$, while that the desired signals for the users in CHV2 served region are $\left\{x_{1}, x_{3}\right\}$. In this assumption, we consider that CHV1 will only decode $\left\{x_{1}, x_{2}\right\}$, and CHV2 will decode $\left\{x_{1}, x_{2}, x_{3}\right\}$ following NOMA decoding principle with the received SNRs for $x_{2}$ and $x_{3}$ as

$$
\gamma_{B_{1}}^{\left(x_{2}\right)}=\frac{\left|h_{B_{1}}\right|^{2} a_{2} \rho}{\left|h_{B_{1}}\right|^{2} a_{3} \rho+1},
$$

and

$$
\gamma_{B_{2}}^{\left(x_{2}\right)}=\frac{\left|h_{B_{2}}\right|^{2} a_{2} \rho}{\left|h_{B_{2}}\right|^{2} a_{3} \rho+1}, \gamma_{B_{2}}^{\left(x_{3}\right)}=\left|h_{B_{2}}\right|^{2} a_{3} \rho .
$$

It worth noting that, only decode the desired signals in CHVs is resealable and practical. Because of that, on one hand the undesired signals will not be forwarded to the vehicles, on the other hand, it may limit the system performance due to the overall performance is restricted by the poor channel coefficient. During the second time slot, CHVs forward new superposition coded signals to the users in the corresponding served region. For simplicity, we assume that the signal $\sqrt{b_{1}} x_{1}+\sqrt{b_{2}} x_{2}$ will be forwarded by CHV1 with $b_{1}+b_{2}=1$, and $\sqrt{c_{1}} x_{1}+\sqrt{c_{2}} x_{3}$ will be forwarded by CHV2 with $c_{1}+c_{2}=1$, where $b_{i}$ and $c_{i}$, for $i \in\{1,2\}$, are new power allocation factors. In this time slot, without loss of generality, there are two kinds of situations that are: 1) V1 is in the overlap region, 2) V1 is not in the overlap region. In following subsections, we will discuss these two situations in details for a better readability.

\subsection{Overlap case}

In this case, the V1 is in the overlap region, leading to that it will receive two superposition coded signals from both of CHV1 and CHV2. And hence, the received signals at V1 can be written as 


$$
\mathcal{Y}_{\mathrm{ol}}^{(V 1)}=\left(\sqrt{b_{1}} h_{v_{1}, U 1}+\sqrt{c_{1}} h_{v_{2}, U 1}\right) \sqrt{P_{C}} x_{1}+\sqrt{b_{2} P_{C}} h_{v_{1}, U 1} x_{2}+\sqrt{c_{2} P_{C}} h_{v_{2}, U 1} x_{3}+n_{U 1}
$$

where $P_{C}$ is the transmit power at CHV1 and CHV2, while $n_{U 1}$ is the AWGN at V1. We further consider that MRC is adopted as V1 to further improve the system performance, therefore, the effective received SNRs for $x_{1}, x_{2}$ and $x_{3}$ can be respectively presented as

$$
\begin{aligned}
& \gamma_{V 1}^{\left(x_{1}\right)}=\frac{\left|h_{v_{1}, U_{1}}\right|^{2} b_{1} \rho}{\left|h_{v_{1}, U_{1}}\right|^{2} b_{2} \rho+1}+\frac{\left|h_{v_{2}, U_{1}}\right|^{2} c_{1} \rho}{\left|h_{v_{2}, U_{1}}\right|^{2} c_{2} \rho+1}, \\
& \gamma_{V 1}^{\left(x_{2}\right)}=\left|h_{v_{1}, U_{1}}\right|^{2} b_{2} \rho \text { and } \gamma_{\mathrm{V} 1}^{\left(\mathrm{x}_{3}\right)}=\left|\mathrm{h}_{\mathrm{v}_{2}, \mathrm{U}_{1}}\right|^{2} \mathrm{c}_{2} \rho,
\end{aligned}
$$

where $\rho=\frac{P_{C}}{N_{0}}$. From Eq. (10), it is easy to see that, if the channels $h_{v_{1}, U_{1}}$ and $h_{v_{2}, U_{1}}$ are very weak, that may results in a serious performance limitation when calculating ergodic SR. Therefore, in our proposed scheme, the SIC is only used to decode the desired signal, i.e., only decode $x_{1}$. In the meanwhile that, the received signals at V2 and V3 are given as

$$
\mathcal{Y}_{\mathrm{ol}}^{(V 2)}=\sqrt{b_{1} P_{C}} h_{v_{1}, U 2} x_{1}+\sqrt{b_{2} P_{C}} h_{v_{1}, U 2} x_{2}+n_{U 2}
$$

with the corresponding received SNRs for $x_{1}$ and $x_{2}$ as $\gamma_{V 2}^{\left(x_{1}\right)}=\frac{\left|h_{v_{1}}, U_{2}\right|^{2} b_{1} \rho}{\left|h_{v_{1}}, u_{2}\right|^{2} b_{2} \rho+1}$ and $\gamma_{V 2}^{\left(x_{2}\right)}=\left|h_{v_{1}, U_{2}}\right|^{2} b_{2} \rho$, and

$$
\mathcal{Y}_{\mathrm{ol}}^{(V 3)}=\sqrt{c_{1} P_{C}} h_{v_{2}, U 3} x_{1}+\sqrt{c_{2} P_{C}} h_{v_{2}, U 3} x_{3}+n_{U 3},
$$

with the corresponding received SNRs for $x_{1}$ and $x_{3}$ as $\gamma_{V 3}^{\left(x_{1}\right)}=\frac{\left|h_{\nu_{2}}, U_{3}\right|^{2} c_{1} \rho}{\left|h_{v_{2}}, U_{3}\right|{ }^{2} c_{2} \rho+1}$ and $\gamma_{V 3}^{\left(x_{3}\right)}=\left|h_{v_{2}, U_{3}}\right|^{2} c_{2} \rho$, where $n_{U i}$, for $i \in\{1,2\}$, denotes the AWGN at $V_{i}$. Remarkably, even that $x_{1}$ is not the desired signal for V2 and V3, however, in order to strictly follow the NOMA decoding principle, $x_{2}$ and $x_{3}$ should be decoded after $x_{1}$ decoded by adopting SIC. With above observations, the overall effective received SNRs for $x_{1}, x_{2}$ and $x_{3}$ in our proposed overlap system can be respectively written as

$$
\begin{aligned}
& \gamma^{\left(x_{1}\right)}=\min \left(\gamma_{B_{1}}^{\left(x_{1}\right)}, \gamma_{B_{2}}^{\left(x_{1}\right)}, \gamma_{V 1}^{\left(x_{1}\right)}, \gamma_{V 2}^{\left(x_{1}\right)}, \gamma_{V 3}^{\left(x_{1}\right)}\right), \\
& \gamma^{\left(x_{2}\right)}=\min \left(\gamma_{B_{1}}^{\left(x_{2}\right)}, \gamma_{B_{2}}^{\left(x_{2}\right)}, \gamma_{V 2}^{\left(x_{2}\right)}\right), \\
& \gamma^{\left(x_{3}\right)}=\min \left(\gamma_{B_{2}}^{\left(x_{3}\right)}, \gamma_{V 3}^{\left(x_{3}\right)}\right) .
\end{aligned}
$$

\subsection{Non-overlap case}

In this case, we assume that V1 is in the CHV1 serviceable region, and out of service from CHV2. Since the receptions for CHV1, CHV2, V2 and V3 are similar to the ones in overlap situation, we omit them. In this manner, it will only receive the signal transmitted from CHV1 as:

$$
\mathcal{Y}_{\text {non }}^{(V 1)}=\sqrt{b_{1}} h_{v_{1}, U 1} \sqrt{P_{C}} x_{1}+\sqrt{c_{2} P_{C}} h_{v_{2}, U 1} x_{2}+n_{U 1},
$$

with the corresponding received SNRs for $x_{1}$ and $x_{2}$ as 


$$
\xi_{V 1}^{\left(x_{1}\right)}=\frac{\left|h_{v_{1}, U_{1}}\right|^{2} b_{1} \rho}{\left|h_{v_{1}, U_{1}}\right|^{2} b_{2} \rho+1},
$$

and

$$
\xi_{V 1}^{\left(x_{2}\right)}=\left|h_{v_{1}, U_{1}}\right|^{2} b_{2} \rho .
$$

Similar to the overlap case, $x_{2}$ will not be decoded. Therefore, the overall effective received SNRs for $x_{1}, x_{2}$ and $x_{3}$ in the non-overlap system can be respectively written as

$$
\begin{aligned}
& \xi^{\left(x_{1}\right)}=\min \left(\gamma_{B_{1}}^{\left(x_{1}\right)}, \gamma_{B_{2}}^{\left(x_{1}\right)}, \xi_{V 1}^{\left(x_{1}\right)}, \gamma_{V 2}^{\left(x_{1}\right)}, \gamma_{V 3}^{\left(x_{1}\right)}\right), \\
& \xi^{\left(x_{2}\right)}=\gamma^{\left(x_{2}\right)} \text { and } \xi^{\left(x_{3}\right)}=\gamma^{\left(x_{2}\right)} .
\end{aligned}
$$

In conventional NOMA scheme, after receptions, all the CHVs will immediately decode the received signals, i.e., $x_{1}, x_{2}$ and $x_{3}$, and then forward the signals to vehicles. On the other hand, the V1 and V2 will also decode the all received signals.

\section{Ergodic sum-rate analysis for the proposed NOMA-IoV networks}

In this section, the overall performance of the ergodic SR and outage probability for our proposed NOMA-IoV networks are analyzed to show the advantages of our proposed schemes, where the V1 are with different locations, i.e., in the overlap region and non-overlap region.

\subsection{Overlap case}

Letting $\beta_{\mu} \triangleq\left|h_{\mu}\right|^{2}$, for $\mu \in\left\{B_{1}, B_{2},\left(v_{1}, U_{1}\right),\left(v_{1}, U_{2}\right),\left(v_{2}, U_{1},\right)\left(v_{2}, U_{2}\right)\right\}$, we have

$$
\mathcal{X}=\min \left(\frac{\beta_{B_{1}} a_{1} \rho}{2 \beta_{B_{1}} a_{2} \rho+1}, \frac{\beta_{B_{2}} a_{1} \rho}{2 \beta_{B_{2}} a_{2} \rho+1}, \frac{\beta_{v_{1}, U_{1}} b_{1} \rho}{\beta_{v_{1}, U_{1}} b_{2} \rho+1}+\frac{\beta_{v_{2}, U_{1}} c_{1} \rho}{\beta_{v_{2}, U_{1}} c_{2} \rho+1}, \frac{\beta_{v_{1}}, U_{2} b_{1} \rho}{\beta_{v_{1}}, U_{2} b_{2} \rho+1}, \frac{\beta_{v_{2}, U_{3}} b_{1} \rho}{\beta_{v_{2}, U_{3}} b_{2} \rho+1}\right) .
$$

Based on above equation, the complementary cumulative distribution function (CCDF) of $\mathcal{X}$ can be obtained from

$$
\begin{gathered}
\bar{F}_{X}(x)=\operatorname{Pr}\left\{\frac{\beta_{B_{1}} a_{1} \rho}{\beta_{B_{1}}\left(a_{2}+a_{3}\right) \rho+1}>x, \frac{\beta_{B_{2}} a_{1} \rho}{\beta_{B_{2}}\left(a_{2}+a_{3}\right) \rho+1}>x, \frac{\beta_{\nu_{1}, U_{2}} b_{1} \rho}{\beta_{v_{1}}, U_{2} b_{2} \rho+1}>x,\right. \\
\left.\frac{\beta_{\nu_{1}, U_{1}} b_{1} \rho}{\beta_{v_{1}, U_{1}} b_{2} \rho+1}+\frac{\beta_{v_{2}, U_{1}} c_{1} \rho}{\beta_{v_{2}, U_{1}} c_{2} \rho+1}>x, \frac{\beta_{\nu_{2}, U_{3}} b_{1} \rho}{\beta_{v_{2}, U_{3}} b_{2} \rho+1}>x\right\} .
\end{gathered}
$$

Noting that the CCDF of $\beta_{\mu}=e^{-\frac{x}{\alpha_{\mu}}}$, when $x<\min \left\{\frac{a_{1}}{a_{2}+a_{3}}, \frac{b_{1}}{b_{2}}\right\}$, (17) can be approximately represented as

$$
\begin{aligned}
\bar{F}_{X}(x) & \bar{F}_{B_{1}}\left(\frac{x}{\left(a_{1}-\left(a_{2}+a_{3}\right) x\right) \rho}\right) \bar{F}_{B_{2}}\left(\frac{x}{\left(a_{1}-\left(a_{2}+a_{3}\right) x\right) \rho}\right) \bar{F}_{v_{1}, U_{2}}\left(\frac{x}{\left(b_{1}-b_{2} x\right) \rho}\right) \\
& \times \bar{F}_{v_{2}, U_{3}}\left(\frac{x}{\left(b_{1}-b_{2} x\right) \rho}\right) \bar{F}_{v_{1}, U_{1}}\left(\frac{x-\frac{c_{1}}{c_{2}}}{\left(b_{1}-b_{2}\left(x-\frac{c_{1}}{c_{2}}\right)\right) \rho}\right) \\
= & e^{-\frac{x}{\left(a_{1}-\left(a_{2}+a_{3}\right) x\right) \rho}\left(\frac{1}{\alpha_{B_{1}}}+\frac{1}{\alpha_{B_{2}}}\right)-\frac{x}{\left(b_{1}-b_{2} x\right) \rho}\left(\frac{1}{\alpha_{v_{1}}, U_{2}}+\frac{1}{\alpha_{\nu_{2}}, U_{3}}\right)-\frac{x c_{2}-c_{1}}{\left(b_{1} c_{2}-b_{2}\left(x c_{2}-c_{1}\right)\right) \rho \alpha_{v_{1}, U_{1}}}}
\end{aligned}
$$


where, for the first term, we approximate $\frac{\beta_{v_{1}}, u_{1} b_{1} \rho}{\beta_{v_{1}}, u_{1} b_{2} \rho+1}+\frac{\beta_{v_{2}}, u_{1} c_{1} \rho}{\beta_{v_{2}}, u_{1} c_{2} \rho+1} \sim \frac{\beta_{v_{1}}, u_{1} b_{1} \rho}{\beta_{v_{1}}, u_{1} b_{2} \rho+1}+\frac{c_{1}}{c_{2}}$ by considering the high transmit SNR, since it is difficult to obtain the exact closed-form expression of $\gamma_{V_{1}}^{\left(x_{1}\right)}$.

Denote $\mathcal{Y}=\min \left(\gamma_{B_{1}}^{\left(x_{2}\right)}, \gamma_{B_{2}}^{\left(x_{2}\right)}, \gamma_{V 2}^{\left(x_{2}\right)}\right)$, and hence the CCDF of $\mathcal{Y}$ for $x_{2}$ can be obtained from

$$
\begin{aligned}
\bar{F}_{Y}(y) & =\operatorname{Pr}\left(\frac{\beta_{B_{1}} a_{2} \rho}{\beta_{B_{1}} a_{3} \rho+1}>y, \frac{\beta_{B_{2}} a_{2} \rho}{\beta_{B_{1}} a_{3} \rho+1}>y, \beta_{\nu_{1}, U_{2}} b_{2} \rho>y\right) \\
& =e^{-\frac{y}{\left(a_{2}-a_{3} y\right) \rho \alpha_{B_{1}}}-\frac{y}{\left(a_{2}-a_{3} y\right) \rho \alpha_{B_{2}}}-\frac{y}{b_{2} \rho \alpha_{\nu_{1}, U_{2}}}} .
\end{aligned}
$$

On the other hand, denoting $\mathcal{Z}=\min \left(\gamma_{B_{2}}^{\left(x_{3}\right)}, \gamma_{V 3}^{\left(x_{3}\right)}\right)$, we have the closed-form expression for $\mathcal{Z}$ as:

$$
\begin{aligned}
\bar{F}_{Z}(z) & =\operatorname{Pr}\left(\beta_{B_{2}} a_{3} \rho>z, \beta_{\nu_{2}}, U_{3} c_{2} \rho>z\right) \\
& =e^{-\frac{z}{a_{3} \rho \alpha_{B_{2}}}-\frac{z}{c_{2} \rho \alpha_{\nu_{2}}, U_{3}}} .
\end{aligned}
$$

After introducing the equalities

$$
\int_{0}^{\infty} \log _{2}(1+x) f_{X}(x) d x=\frac{1}{\ln 2} \int_{0}^{\infty} \frac{1-F(x)}{1+x} d x,
$$

and

$$
\int_{0}^{u} \frac{e^{-\mu x} d x}{x+\beta}=e^{\mu \beta}[\operatorname{Ei}(-\mu \mathrm{u}-\mu \beta)-\operatorname{Ei}(-\mu \beta)],
$$

[32, Eq. (3.352.1)], where Ei $(\cdot)$ means the exponential integral function. Since that the closed-form expression of ergodic rate for $x_{1}$ is quite difficult, we focuss on the high transmit SNR case, i.e., $\rho \gg 1$. In addition, for simplicity, we further assume that $a_{1}<b_{1}$ due to that there exists three signals in $\mathcal{X}$ and only two in $\mathcal{Y}$. Therefore, when $x<\min \left\{\frac{a_{1}}{a_{2}+a_{3}}, \frac{b_{1}}{b_{2}}, \frac{b_{1}}{b_{2}}+\frac{c_{1}}{c_{2}}\right\}=\min \left\{\frac{a_{1}}{1-a_{1}}, \frac{b_{1}}{1-b_{1}}\right\}=\frac{a_{1}}{1-a_{1}}$, the ergodic rate for $x_{1}$ in the overlap case can be respectively written as

$$
\begin{aligned}
& C_{\mathrm{ol}}^{\left(x_{1}\right)} \sim \frac{1}{2 \ln 2} \int_{0}^{\frac{a_{1}}{1-a_{1}}} \frac{e^{-\frac{x \mathcal{A}}{\left(a_{1}-\left(1-a_{1}\right) x\right)}-\frac{x \mathcal{B}}{\left(b_{1}-b_{2} x\right) \rho}}}{1+x} d_{x}
\end{aligned}
$$

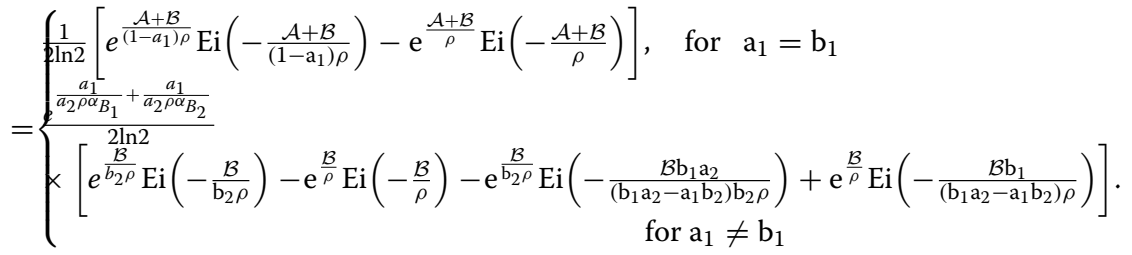

where $\mathcal{A}=\frac{1}{\alpha_{B_{1}}}+\frac{1}{\alpha_{B_{2}}}$ and $\mathcal{B}=\frac{1}{\alpha_{v_{1}}, U_{1}}+\frac{1}{\alpha_{v_{1}}, U_{2}}+\frac{1}{\alpha_{v_{2}}, u_{3}}$, for the case $x>\frac{a_{1}}{1-a_{1}}, C_{\mathrm{ol}}^{\left(x_{1}\right)}=0$ always holds. For $x_{2}$, we have 


$$
\begin{aligned}
C_{\mathrm{ol}}^{\left(x_{2}\right)} & \sim \frac{1}{2 \ln 2} \int_{0}^{\frac{a_{2}}{a_{3}}} \frac{e^{-\frac{y \mathcal{A}}{\left(a_{2}-a_{3} y\right) \rho}-\frac{y}{b_{2} \rho \alpha_{v_{1}, U_{2}}}}}{1+y} d_{y} \\
& \sim \frac{1}{2 \ln 2} e^{\frac{a_{2} \mathcal{A}}{a_{3} \rho}+\frac{1}{b_{2} \rho \alpha_{v_{1}}, U_{2}}}\left[\operatorname{Ei}\left(\frac{1}{\mathrm{~b}_{2} \rho \alpha_{\mathrm{v}_{1}, \mathrm{U}_{2}}}\right)-\operatorname{Ei}\left(\frac{\mathrm{a}_{2}+\mathrm{a}_{3}}{\mathrm{a}_{3} \mathrm{~b}_{2} \rho \alpha_{\mathrm{v}_{1}, \mathrm{U}_{2}}}\right)\right],
\end{aligned}
$$

where (22) follows the condition $x<\frac{a_{2}}{a_{3}}$, otherwise $C_{\mathrm{ol}}^{\left(x_{2}\right)}=0$. In the meanwhile, the ergodic rate in closed form for $x_{3}$ can be obtained from

$$
\begin{aligned}
C_{\mathcal{D}_{2}}^{\left(x_{2}\right)} & =\frac{1}{2 \ln 2} \int_{0}^{\infty} \frac{e^{-\frac{z}{a_{3} \rho \alpha_{B_{2}}}-\frac{z}{c_{2} \rho \alpha_{v_{2}}, U_{3}}}}{1+z} d_{z} \\
& =\frac{1}{2 \ln 2} e^{\frac{1}{a_{3} \rho \alpha_{B_{2}}}+\frac{1}{c_{2} \rho \alpha_{v_{2}}, U_{3}}} \operatorname{Ei}\left(-\frac{1}{\mathrm{a}_{3} \rho \alpha_{\mathrm{B}_{2}}}-\frac{1}{\mathrm{c}_{2} \rho \alpha_{\mathrm{v}_{2}, \mathrm{U}_{3}}}\right) .
\end{aligned}
$$

Finally, the ergodic SR for the proposed overlap scheme can be expressed as

$$
C_{\text {sum }} l=C_{\mathrm{ol}}^{\left(x_{1}\right)}+C_{\mathrm{ol}}^{\left(x_{2}\right)}+C_{\mathrm{ol}}^{\left(x_{3}\right)} .
$$

For non-overlap case, it is clear that the analysis results for the non-overlap case will follow the received SNRs as shown in $\xi^{\left(x_{1}\right)}, \xi^{\left(x_{2}\right)}$ and $\xi^{\left(x_{3}\right)}$. There is only one difference between the proposed two cases that are $\xi^{\left(x_{1}\right)_{V} 1}$ and $\gamma^{\left(x_{1}\right)_{V} 1}$. Therefore, for simplicity and better readability, in the manuscript, we omitted them.

\section{Results and discussion}

\subsection{Numerical results}

In this section, we show the numerical results in terms of ergodic SR to demonstrate the performance of the proposed NOMA-IoV system. The numerical results are averaged over 100, 000 channel realizations with Rayleigh fading. In following figures, we use "Simulation" to denote the simulation results, while the "Analysis" is used to presented the analytical results. The simulation set up is given as follows: $\alpha_{v_{1}, U_{1}}=1, \alpha_{v_{1}, U_{2}}=10$, $\alpha_{v_{2}, U_{1}}=1, \alpha_{v_{2}, U_{3}}=10, a_{1}=0.7, a_{2}=0.2, a_{3}=0.1, b_{1}=0.7, b_{2}=0.3$ and $c_{1}=c_{2}=0.5$

Figure 2 depicts the ergodic SR performance of our proposed NOMA-IoV scheme versus the transmit SNR, where two channel coefficient setups are considered: (1) $\alpha_{B_{1}}=1, \alpha_{B_{2}}=10$; (2) $\alpha_{B_{1}}=10, \alpha_{B_{2}}=1$. The motivations for these two groups dependent on the effect of CHV1 and CHV2, since that the poor channel quality will limit to a significantly performance limitation. As shown in Fig. 2, it is easy to see that our proposed scheme for both overlap and non-overlap cases overwhelm that of the conventional NOMA scheme, due to that the undesired signals for the V2 and V2 will not be decoded. With the growing SNRs, our proposed scheme illustrate more obvious advantages. In addition, it is also shown that, for our proposed NOMA-IoV scheme, the overlap case is better than the non-overlap case at the low SNR region, and these two cases are almost same at the high SNR region. This is reasonable, because of that, when $\rho$ goes to infinity, the influence of the channel quality to the system performance will be weak replaced by the SNR. Moreover, it is clear that the simulation results tightly fit the analysis results especially in the high SNR region, which verifies our theoretical analysis. It is worth noting that, we approximate Eqs. (16) and (17) by considering the high transmit 


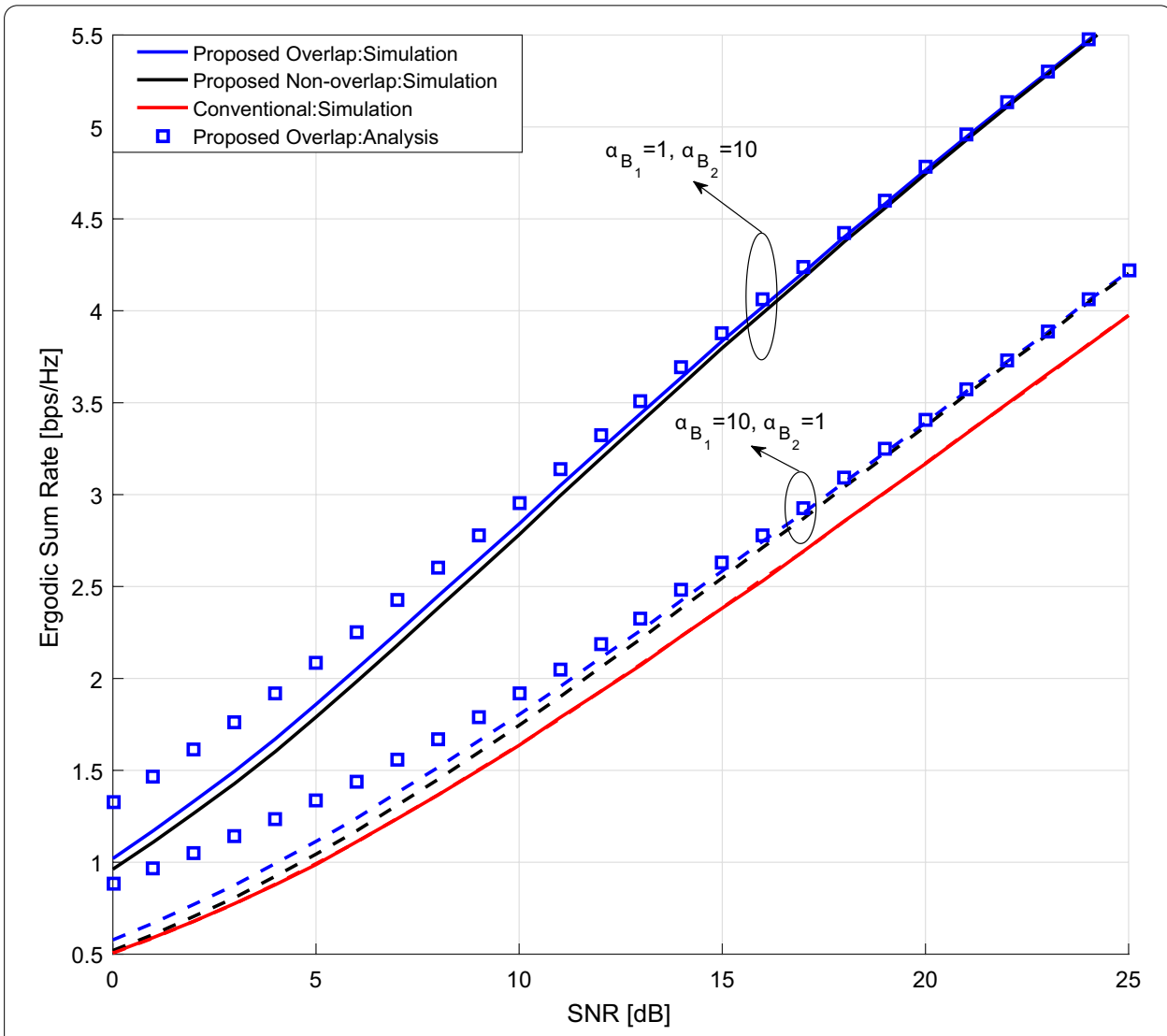

Fig. 2 The ergidic SR for our proposed NOMA-loV system including overlap and non-overlap cases compared with conventional NOMA scheme versus the transmit SNR

SNR, since it is difficult to obtain the exact closed-form expression of $\gamma_{V_{1}}^{\left(x_{1}\right)}$. On one hand, the approximation is only used partial of Eq. (15) as $\frac{\beta_{v_{2}, U_{1}} c_{1} \rho}{\beta_{v_{2}}, U_{1} c_{2} \rho+1} \sim \frac{c_{1}}{c_{2}}$. On the other hand, as shown in numerical results, there is a good match between the simulation and analysis results, especially at high SNR region. Therefore, these illustrates that the performance loss provided from the approximation is negligible.

Figure 3 shows the ergodic SR performance of the overlap case for our proposed NOMA-IoV scheme with respect to the average channel power $\alpha_{\zeta}$, for $\varsigma \in\left\{\left(v_{1}, U_{1}\right),\left(v_{1}, U_{2}\right), B_{1}, B_{2}\right\}$. In these subfigures, we also consider the different SNR conditions, i.e., $\rho=\{10,15,20,25\} \mathrm{dB}$. All the subfigures depict that, even that $\alpha_{B_{1}}$ has the maximum impact on the system, the ergodic SR performance with respect to it are always the smallest from low to high SNRs. This is reasonable, because of that, with Eq. (15), it is easy to see if the channel coefficient is smaller, the results for the received SNR will change fast. According to the simulation set up, we have defined $\alpha_{B_{1}}$ as a smallest one for another one is greater (without loss of generality). Particularly, the ergodic sum-rate has varying degrees of improvements for all the cases. In addition, the $\alpha_{\nu_{1}}, U 1$ provides the maximum ergodic SR in every situations, especially at low SNR region. For high SNR case, $\alpha_{\zeta}$ shows similar results, except $\alpha_{B_{1}}$.

Figure 4 illustrates the ergodic SR performance of the overlap case for our proposed NOMA-IoV system versus the power allocation factors $a_{1}$ and $b_{1}$, where we have fixed 


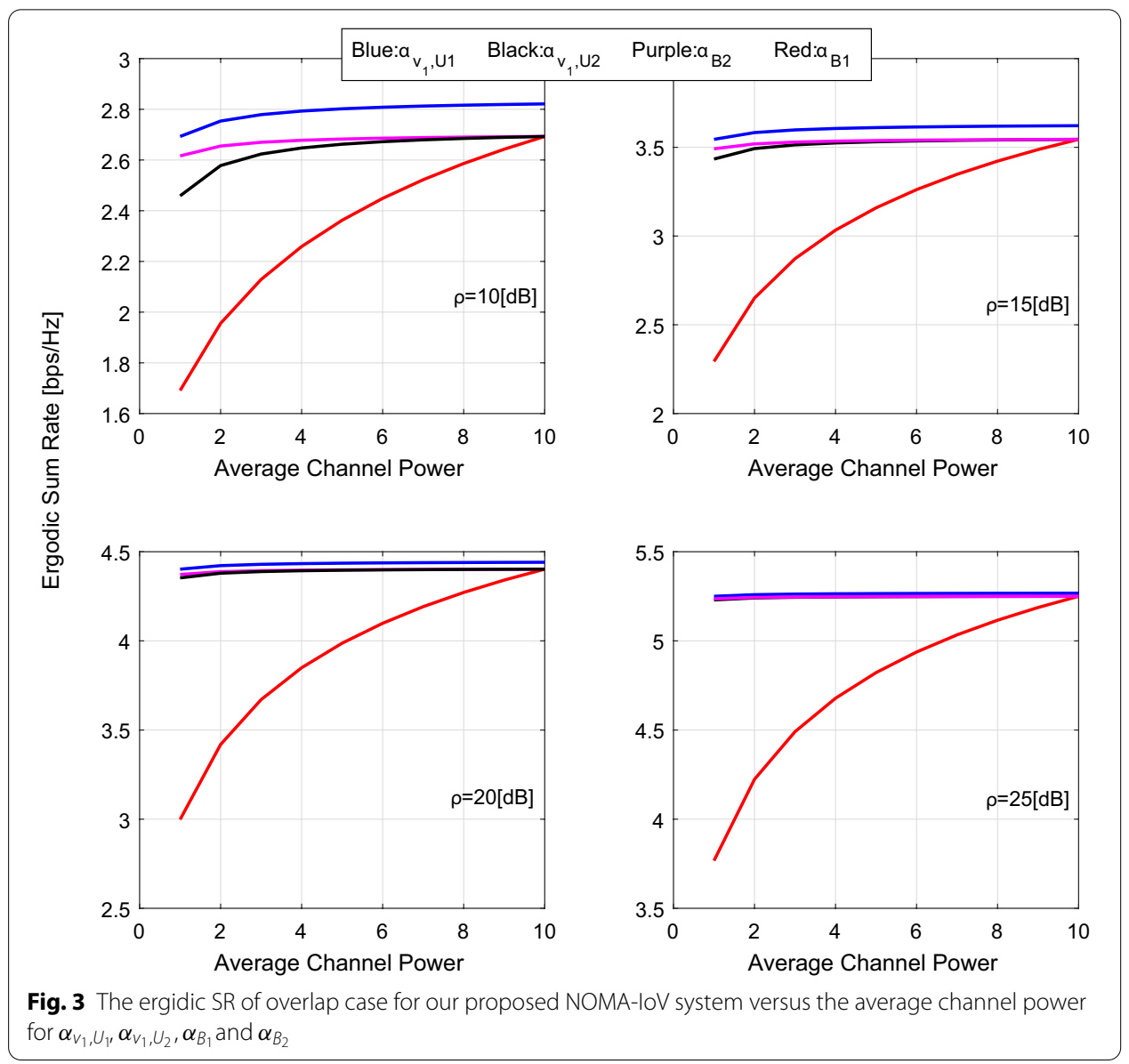

$\alpha_{B 1}=1, \alpha_{B 2}=10$, and $a_{3}=0.01$. Different transmit SNRs are considered including $\rho=\{5,10,15,20\} \mathrm{dB}$, respectively. Clearly, for all subfigures, the maximum ergodic SR will be achieved for a value of $a_{1}$ closed to " 1 " while $b_{1}$ closed to " 0.5 ", due to that, for the greater $a_{1}$, similar $b_{1}$ and $b_{2}$, i.e., $\left\{b_{1}, b_{2}\right\}$ close to 0.5 with $b_{1}+b_{2}=1$, the maximum received SNRs in Eq. (15) will be achieved. To sum up, the ergodic SR of the proposed NOMA-IoV provides an outstanding advantage over the conventional NOMA one, and the overlap case achieves better performance than the non-overlap case at a low SNR region.

\subsection{Discussion}

For the conventional NOMA scheme, after receptions from BS, CHV1 and CHV2 will simultaneously decode the received signals, and then forward them to V1, V2 and V3. However, if the receiving node decode the signal transmitted via a very poor channel, and these decoded signals are not the the demands (the desired signal) of V1, V2 and V3, that will lead to a significantly performance limitation. Moreover, in conventional NOMA scheme, V1, V2 and V3 will also decode all the received signal without considering the demands, resulting in a further performance loss. Therefore, different from conventional works, we focus on the demands of vehicles to avoid the 


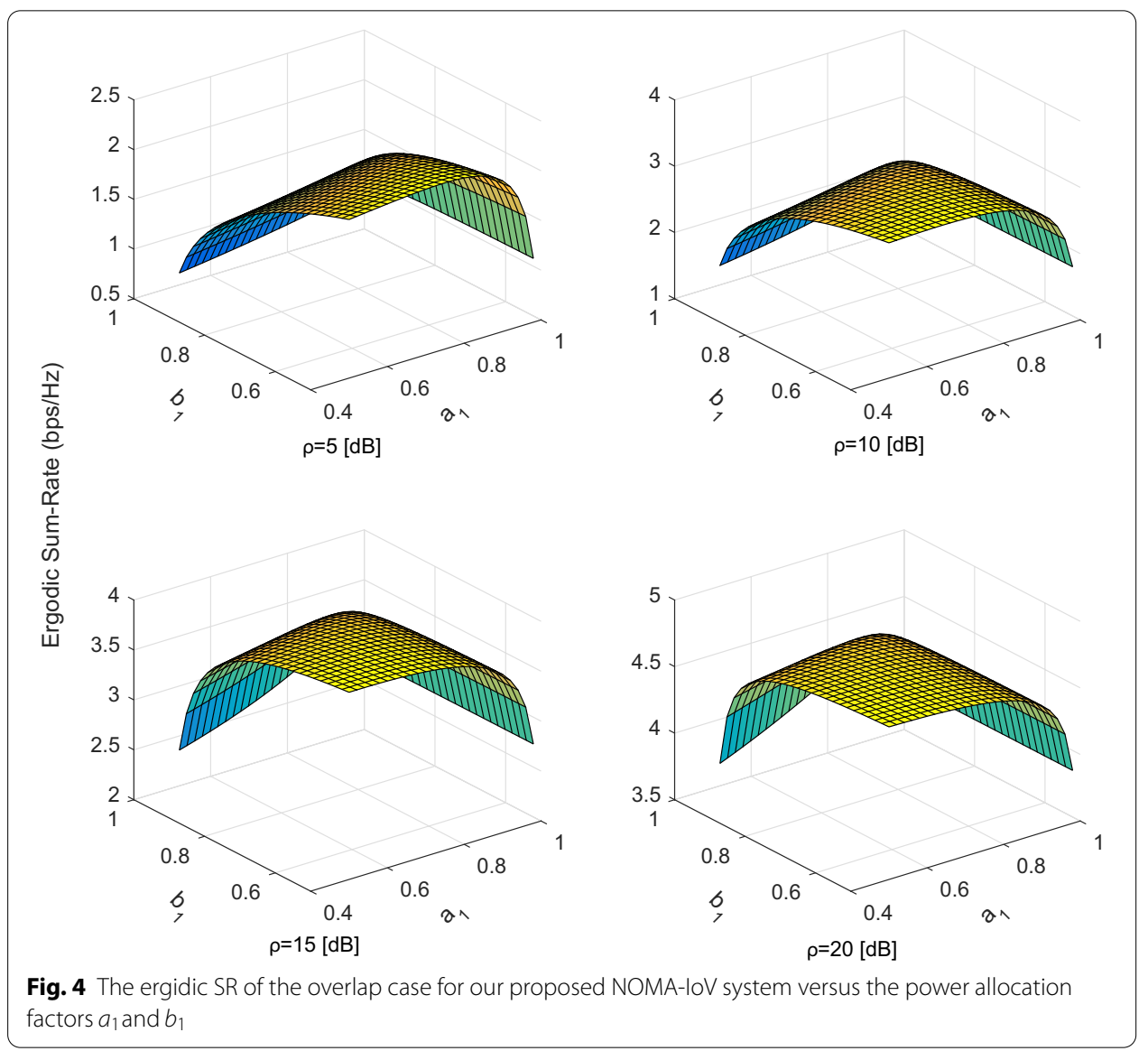

Table 1 Decode elements of the proposed overlap and conventional NOMA schemes

\begin{tabular}{lll}
\hline Item & Conventional NOMA & Proposed NONA-IoV \\
\hline CHV1 & $x_{1}, x_{2}, x_{3}$ & $x_{1}, x_{2}$ \\
CHV2 & $x_{1}, x_{2}, x_{3}$ & $x_{1}, x_{2}, x_{3}$ \\
V1 & $x_{1}, x_{2}, x_{3}$ & $x_{1}$ \\
V2 & $x_{1}, x_{2}$ & $x_{1}, x_{2}$ \\
V3 & $x_{1}, x_{3}$ & $x_{1}, x_{3}$ \\
\hline
\end{tabular}

disadvantages discussed above. Finally, the decoding elements for our proposed overlap scheme and conventional NOMA scheme are respectively shown as Table 1 in details.

\section{Conclusions}

In this paper, a novel NOMA-IoV scheme was investigated with different vehicle locations considering the overlap and non-overlap region for CHVs. Different to conventional NOMA schemes, the CHVs only decoded the desired signals for the vehicles in next hop to avoid the system performance loss. Adopting MRC, the vehicle in overlap region received signals from $\mathrm{CHV} 1$ and $\mathrm{CHV} 2$ resulting in a significantly ergodic rate improvement. Moreover, the theoretical derivations have been shown to highly agree 
with the simulation results, especially at the high SNR region. By means of the numerical results, our proposed NOMA-IoV scheme has been shown to exhibit a better SR behavior than the conventional NOMA scheme. Moreover, in our proposed scheme, the ergodic SR for the overlap case is better than the non-overlap case at the low SNR, and is similar at high SNR. Our future concerns will be the joint optimization on the power allocations at BS and CHVs.

\author{
Abbreviations \\ (NOMA): Non-orthogonal multiple access; (OMA): Orthogonal multiple access; (SIC): Successive interference cancellation; \\ (MRC): Maximum ratio combining; (SR): Sum-rate; (PA): Power allocation; (TDMA): Time-division multiple access; (CSI): \\ Channel state information; (DF): Decode-and-forward; (AWGN): Additive white Gaussian noise; (BS): Base station; (CHV): \\ Cluster head vehicle.
}

Acknowledgements

The author would like to thank the Editor and reviewers for their valuable and insightful comments.

\title{
Authors' contributions
}

J. Gu, W. Duan, and G. Zhang conceived and designed the study. J. Gu and W. Duan performed the simulations. J. Gu and G. Zhang wrote the paper. W. Duan, and G. Zhang reviewed and edited the manuscript. All authors read and approved the final manuscript.

\section{Funding}

This work was supported in part by the National Natural Science Foundation of China under Grant Grant 61971245 and Grant 61801249, Class C project funded by the 16th batch of 'six talent peaks' high level talent selection and training in Jiangsu Province, grant number XYDXX-245, "sponsored by Qing Lan Project in Jiangsu Province" and "Supported by the training object of the 6th "521 High-level Talents Training Project" in Lianyungang ".

\section{Availability of data and materials}

The authors declare that all the data and materials in this manuscript are available. In addition, a MATLAB tool has been used to simulate our concept.

\section{Declaration}

\section{Competing interests}

The authors declare that they have no competing interests.

\section{Author details}

${ }^{1}$ School of Information Science and Technology, Nantong University, Nantong 226000, China. ${ }^{2}$ Kangda College of Nanjing Medical University, Lianyungang 222000, China. ${ }^{3}$ Institute of North China Sea Offshore Engineering Survey, Ministry of Natural Resources North Sea Bureau, Qingdao 266000, China.

Received: 21 November 2020 Accepted: 9 March 2021

Published online: 23 March 2021

\section{References}

1. D. Mohr et al., Automotive revolution and persepective towards 2030. Auto Technol. Rev. (2016)

2. E.A. Feukeu, S.M. Ngwira, T. Zuva, Doppler shift signature for bpsk in a vehicular network: IEEE 802.11 p, in Proceedings of the IEEE ICMA, Beijing, China (2015), pp. 1744-1749

3. Z. Lv et al., Solving the security problem of intelligent transportation system with deep learning. IEEE Trans. Intell. Transp. Syst. (to appear) (2020)

4. Z. Lv et al., Interaction of edge-cloud computing based on SDN and NFV for next generation IoT. IEEE Internet Things J. 7(7), 5706-5712 (2020)

5. L. Hu et al., Unified device-to-device communications for low latency and high reliable vehicle-to-X services, in Proceedings of the IEEE VTC-Spring, Nanjing, China (2016)

6. W. Duan et al., Emerging technologies for 5G-loV networks: applications, trends and opportunities. IEEE Netw. 34(5), 283-289 (2020)

7. S.. Chen, Jinling Hu, Y.. Shi, L.. Zhao, Networking technology, standards and applications of LTE-V2X. Telecom Sci. 34(4), 1-11 (2008)

8. IEEE Standard for Information Technology-Telecommunications and Information Exchange Between Systems-Local and Metropolitan Area Networks-Specific Requirements-Part 3: Carrier Sense Multiple Access with Collision Detection (CSMA/CD) Access Method and Physical Layer Specifications-Data Terminal Equipment (DTE) Power Via Media Dependent Interface (MDI), in IEEE Std 802.3af-2003 (2003), pp. 1-133

9. G. Araniti et al., LTE for vehicular networking: a survey. IEEE Commun. Mag. 51(5), 148-157 (2015)

10. 3GPP RP-161894, LTE-Based V2X Services (2016)

11. Z. Wu, V. Park, J. Li, Enabling device to device broad-cast for LTE cellular networks. IEEE J. Sel. Areas Commun. 34(1) 58-70 (2016) 
12. C. Wang, F. Haider, X. Gao et al., Cellular architecture and key technologies for $5 \mathrm{G}$ wireless communication networks. IEEE Commun. Mag. 52(2), 122-130 (2014)

13. W. Duan, Y. Ji, J. Hou, B. Zhuo, M. Wen, G. Zhang, Partial-DF full-duplex D2D-NOMA systems for loT with/without an eavesdropper. IEEE Internet Things J. (to appear) (2021)

14. X. Pei et al., Socially-aware joint resource allocation and computation offloading in NOMA-aided energy harvesting massive loT. IEEE Internet Things J. (to appear) (2021)

15. Q.. Li et al., Spatial modulation-aided cooperative NOMA: performance analysis and comparative stud. IEEE J. Sel. Top. Signal Process. 13(3), 715-728 (2019)

16. D. Wan et al., Non-orthogonal multiple access for cooperative communications: challenges, opportunities, and trends. IEEE Wirel. Commun. 25(2), 109-117 (2018)

17. B. Zheng et al., Secure NOMA based two-way relay networks using artificial noise and full duplex. IEEE J. Sel. Areas Commun. 36(7), 1426-1440 (2018)

18. J.G. Andrews et al., What will 5 G be? IEEE J. Sel. Areas Commun. 32(6), 1065-1082 (2014)

19. H. Zheng, H. Li, S. Hou, Z. Song, Joint resource allocation with weighted max-min fairness for NOMA-enabled V2X communications. IEEE Access 6, 65449-65462 (2018)

20. D.W.K. Ng, E. Lo, R. Schober, Energy-efficient resource allocation in OFDMA systems with large numbers of base station antennas. IEEE Trans. Wirel. Commun. 11(9), 3292-3304 (2012)

21. Y. Ji, W. Duan, M. Wen, P. Padidar, J. Li, N. Cheng, P.-H Ho, Spectral efficiency enhanced cooperative device-to-device systems with NOMA. IEEE Trans. Intell. Transp. Syst. (to appear) (2020)

22. B. Di, L. Song, Y. Li, Z. Han, V2X meets NOMA: non-orthogonal multiple access for 5G-enabled vehicular networks. IEEE Wirel. Commun. 24(6), 14-21 (2017)

23. L. Qian, Y. Wu, H. Zhou, X. Shen, Non-orthogonal multiple access vehicular small cell networks: architecture and solution. IEEE Netw. 31(4), 15-21 (2017)

24. S. Guo, X. Zhou, Robust power allocation for NOMA in heterogeneous vehicular communications with imperfect channel estimation, in Proceedings of the IEEE 28th Annual Interantional Symposium Personals, Indoor, Mobile Radio Commun (PIMRC), Montreal, QC, Canada (2017), pp. 1-5

25. C. Chen, B. Wang, R. Zhang, Interference Hypergraph-based Resource Allocation (IHG-RA) for NOMA-integrated V2X networks. IEEE Internet Things J. 6(1), 161-170 (2019)

26. C. Cooper, D. Franklin, M. Ros, F. Safaei, M. Abolhasan, A comparative survey of VANET clustering techniques. IEEE Commun. Surv. Tutor. 19(1), 657-6811 (2017)

27. S.Y. Kannekanti, G.S.P. Nunna, V.K.R. Bobba, A.K. Yadama, A. Elleithy, An efficient clustering scheme in vehicular ad- hoc networks, in Proceedings of the IEEE UEMCON, New York, NY, USA (2017), pp. 282-287

28. A.A. Khan, M. Abolhasan, W. Ni, An evolutionary game theoretic approach for stable and optimized clustering in VANETs. IEEE Trans. Veh. Technol. 67(5), 4501-4513 (2018)

29. J. Wang, K. Liu, K. Xiao, C. Chen, W. Wu, V.C. Lee, S.H. Son, Dynamic clustering and cooperative scheduling for vehicleto-vehicle communication in bidirectional road scenarios. IEEE Trans. Intell. Transp. Syst. 19(6), 1913-1924 (2018)

30. L. Liang, J. Kim, S.C. Jha, K. Sivanesan, G.Y. Li, Spectrum and power allocation for vehicular communications with delayed CSI feedback. IEEE Wirel. Commun. Lett. 6(4), 458-461 (2017)

31. B. Di, L. Song, Y. Li, G.Y. Li, Non-orthogonal multiple access for high-reliable and low-latency V2X communications in 5G systems. IEEE J. Sel. Areas Commun. 35(10), 2383-2397 (2017)

32. M. Abramowitz \& I.A. Stegun, Handbook of Mathematical Functions with Formulas, Graphs, and Mathematical Tables 9th. ed. (Dover, New York, 1970)

\section{Publisher's Note}

Springer Nature remains neutral with regard to jurisdictional claims in published maps and institutional affiliations.

\section{Submit your manuscript to a SpringerOpen ${ }^{\circ}$ journal and benefit from:}

- Convenient online submission

- Rigorous peer review

- Open access: articles freely available online

- High visibility within the field

- Retaining the copyright to your article

Submit your next manuscript at $\boldsymbol{\nabla}$ springeropen.com 